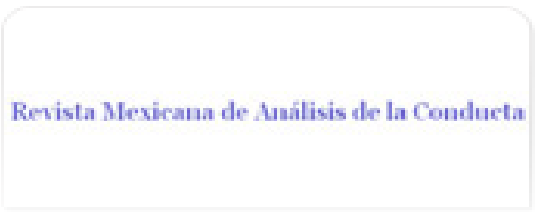

Revista Mexicana de Análisis de la Conducta ISSN: 0185-4534

editora@rmac-mx.org

Sociedad Mexicana de Análisis de la Conducta México

Munguía Rodríguez, Laura Margarita; Martínez Moreno, Alma Gabriela EFECTO DE LA ADMINISTRACIÓN DE NICOTINA SOBRE LA CONDUCTA ALIMENTARIA Revista Mexicana de Análisis de la Conducta, vol. 36, núm. 2, septiembre-noviembre, 2010 Sociedad Mexicana de Análisis de la Conducta Guadalajara, México

Disponible en: http://www.redalyc.org/articulo.oa?id=59314208010

Cómo citar el artículo

- Número completo

- Más información del artículo

Página de la revista en redalyc.org

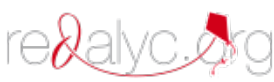

Sistema de Información Científica Red de Revistas Científicas de América Latina, el Caribe, España y Portugal Proyecto académico sin fines de lucro, desarrollado bajo la iniciativa de acceso abierto 
Revista Mexicana de Análisis de la Conducta/

Mexican Journal of Behavior Analysis

Vol. 36, num. 2 Septiembre 2010

Este pdf provisional corresponde a la apariencia del artículo al momento de su aceptación. La versión final del pdf, con la paginación definitiva aparecerá próximamente.

\section{EFECTO DE LA ADMINISTRACIÓN DE NICOTINA SOBRE LA CONDUCTA ALIMENTARIA}

EFFECT OF NICOTINE ADMINISTRATION ON FEEDING BEHAVIOR

Laura Margarita Munguía Rodríguez \& Alma Gabriela Martínez Moreno

Centro de Investigaciones en Comportamiento Alimentario y Nutrición (CICAN) CUSur - Universidad de Guadalajara

Recibido: $\quad$ Abril 14, 2010

Revisado: $\quad$ Junio 21, 2010

Aceptado: Julio 2, 2010

Publicación digital: Julio 25, 2010

URL de la revista: www.journals.unam.mx/rlmc/

ISSN: $\quad 0185-4534$

Este artículo puede ser impreso, descargado y distribuido libremente.

Citarlo de la siguiente manera:

Munguía, L. M., \& Martínez, A. G. (2010) Efecto de la administración de nicotina sobre la conducta alimentaria. Revista Mexicana de Análisis de la Conducta, 36, 2.

Este es un artículo de acceso libre distribuido bajo los términos de la licencia de atribución Creative Comons (http://creativecommons.org/licenses/by/2.0), que permite el uso irrestricto, distribución y reproducción mediante cualquier medio, siempre y cuando se cite apropiadamente el trabajo original.

Esta investigación fue financiada por CONACYT CB-2008/101314. Contribución de los autores: Laura Margarita Munguía Rodríguez ABCDE, Alma Gabriela Martínez Moreno ACDEF; donde: $\mathrm{A}$ = Diseño de Estudio, $\mathrm{B}$ = Recolección de Datos, $\mathrm{C}$ = Interpretación de 
Datos, $\mathrm{D}=$ Preparación de Manuscrito, E = Búsqueda Bibliográfica, F = Gestión de Financiamiento. Correspondencia: Alma Gabriela Martínez Moreno, Centro de Investigaciones en Comportamiento Alimentario y Nutrición, CUSur-Universidad de Guadalajara. Av. Prolongación Colón s/n, Cd. Guzmán, Mpio. de Zapotlán el Grande, Jalisco. CP. 49000. Tel. (01 341) 57522 22, ext. 6127. E-mail:

alma.martinez@cusur.udg.mx

Resumen

La nicotina es una sustancia química que se encuentra en el tabaco y provoca severos cambios en el organismo. Es catalogada como una de las drogas más adictivas. Estudios en ratas han señalado que la administración de nicotina provoca la disminución del consumo de alimento y mantiene bajo el peso corporal. El objetivo de nuestro experimento fue evaluar si la administración de dos diferentes dosis de nicotina tiene efectos sobre el peso corporal y la ingesta de alimento a través de la aplicación de parches transdérmicos. Seis ratas Wistar ingenuas experimentalmente, separadas en tres grupos, fueron expuestas a libre acceso de alimento y agua durante 40 días. Del día 11 al 15 y del día 26 al 30 del experimento, el Grupo 1 fue expuesto a $6 \mathrm{mg}$ de nicotina, el Grupo 2 a $12 \mathrm{mg}$, mientras que el Grupo 3 (control) no recibió ninguna dosis. Los resultados obtenidos sugieren que la nicotina disminuye el consumo de alimento independientemente de la dosis utilizada.

Palabras clave: Nicotina, parche transdérmico, consumo de alimento, peso corporal, ratas

\begin{abstract}
Nicotine is a chemical substance component of tobacco and produces serious changes in the organism. It is catalogued as one of the most addictive drugs. Studies with rats have showed that nicotine administration produces food consumption decrease and favor the maintenance of low body weight. The objective of our experiment was to evaluate if two different doses of nicotine have effects over body weight and food intake through transdermal patches application. Six experimentally naive Wistar rats were separated into three groups and exposed to free access to water and food during 40 days. Day 11 to 15 and 26 to 30 Group 1 was exposed to $4 \mathrm{mg}$ of nicotine, Group 2 to $12 \mathrm{mg}$ and Group 3 (control) did not receive any doses. The results suggest that nicotine decrease food consumption independent of doses used.
\end{abstract}

Key words: Nicotine, trasdermal patches, food consumption, body weight, rats.

\title{
INTRODUCCIÓN
}

La nicotina es una sustancia química que se encuentra en el tabaco. Se ha demostrado de manera experimental que la nicotina puede modificar las preferencias alimentarias, el 
sentido del gusto y alterar los hábitos alimentarios: la ingesta de energía y absorción de nutrientes (Chen, Hansen, Jones, Vlahos, Anderson y Morris 2007; Clarke y Kumar 1984; Grunberg, Bowen, Maycock y Nespor 1985; Grunberg, Bowen y Morse 1984; Grunberg, Popp y Winders 1987; Grunberg, Popp y Winders 1988).

La administración de la nicotina en estudios experimentales se ha realizado mediante vía oral, intraperitonial, o intravenosa. Otros procedimientos de administración de esta sustancia incluyen la exposición al humo de cigarrillos, la implantación quirúrgica subcutánea de aparatos especiales, como las bombas mini osmóticas; y otros implementos más comerciales como los parches transdérmicos. La administración crónica de nicotina por vía oral se utiliza generalmente para estudiar los efectos a largo plazo que provoca esta droga (Nuutinen, 2007). La administración por vía intraperitonial es frecuentemente utilizada por su fácil absorción y acceso a esta vía, representa una de las técnicas más utilizadas en animales de laboratorio como conejos, ratas, ratones, cobayos y perros. Sin embargo, esta técnica provoca altos niveles de estrés en los sujetos debido al tipo de manipulación que requiere (Barber, Fox, Roca y Fresneda, 1987). La administración por vía intravenosa es poco utilizada debido al alto grado de dificultad para dominar la técnica de aplicación (Nuutinen, 2007).

Por otro lado, la exposición al humo de cigarrillos de forma directa es un procedimiento comúnmente utilizado en el laboratorio. Probablemente representa el procedimiento experimental de administración de esta droga más complejo. Los animales se colocan dentro de una cámara de material resistente y transparente. Este equipo es conectado a un aparato con dos tubos especiales para extraer y succionar el humo del cigarrillo (Azevedo, et al. 2010). Otro procedimiento complejo es la implantación de aparatos pequeños dentro del organismo como es el caso de las bombas mini osmóticas. Representan un sistema de implantación subcutánea que administra la nicotina de manera automática y periódica. Este procedimiento es comúnmente utilizado para minimizar el estrés causado al sujeto por las manipulaciones (Nuutinen, 2007). Por otro lado, el parche transdérmico es un implemento poco utilizado en el laboratorio experimental. Contiene un sistema de dispensación transdérmica y la liberación de la droga se realiza de manera progresiva, manteniendo niveles estables durante el tiempo de utilización (Jiménez- Ruiz, et al, 2001). Salin-Pascual, De la Fuente, Galicia-Polo y Drucker-Colin (1995) señalaron que la utilización del parche transdérmico como vía para liberar la nicotina en los organismos representa una herramienta eficaz para mejorar los tratamientos dirigidos a terminar con el hábito de fumar.

Los hallazgos más relevantes sobre el efecto de la administración de nicotina en los organismos se basan principalmente en los daños provocados a la salud. No obstante, se le ha dado poca atención a los estudios sobre la posible participación de la nicotina en la supresión del consumo de alimento. Investigaciones recientes en humanos y animales han encontrado que fumar cigarros o la administración directa de nicotina van acompañados de la disminución del consumo de alimento o comidas altas en calorías y que el cese de la nicotina va seguida por el incremento del consumo de alimento (Grunberg, Bowen y Morse, 1984).

Clarke y Kumar (1984) realizaron un experimento para demostrar que la nicotina puede disminuir el consumo de alimento. Observaron a un grupo de ratas a las que se les administró diariamente $0.4 \mathrm{mg} / \mathrm{kg}$ de nicotina junto a un grupo control que no recibió el tratamiento durante un mes. Durante este periodo los sujetos experimentales disminuyeron 
su consumo de alimento respecto al registrado en el grupo control. Cuando se interrumpió la administración de nicotina no se observaron diferencias en el consumo de alimento de los sujetos experimentales en relación al consumo de los sujetos control. Posteriormente se les administró una solución salina (en sustitución de la nicotina) y observaron que durante este periodo tampoco se presentaron cambios en el consumo de alimento en los dos grupos. Grunberg, Bowen y Morse (1984) señalaron que la administración de nicotina disminuye el consumo de alimento y produce cambios en la preferencia de alimentos. Realizaron dos experimentos: en el primero se suministraron tres diferentes concentraciones de nicotina (4, 8 y $12 \mathrm{mg}$ ) a ratas Sprague Dawley durante dos semanas y se les proporcionó alimento especial para animales de laboratorio. Se observó que cuando las ratas estaban en contacto con la nicotina consumían menos alimento comparado con los periodos de tiempo en los que no estaban en contacto con esta sustancia. Se determinó que a mayor dosis de nicotina, mayor es la disminución en el consumo de alimento dulce. En el segundo experimento se realizó el mismo procedimiento a excepción de que se alargó el periodo de exposición a la nicotina. Obtuvieron los mismos resultados que en el primer experimento, pero no encontraron diferencias significativas. Los autores discutieron la posibilidad de que las ratas muestren mayores modificaciones en su conducta de comer si hay disponibilidad de otros alimentos no insípidos como es el caso de los alimentos dulces o salados.

Grunberg, Bowen, Maycock y Nespor (1985) realizaron un experimento con ratas machos de la cepa Sprague-Dawley durante 17 días a los que se les registró el peso corporal, consumo de alimentos y consumo de agua diariamente. Se administraron dos diferentes concentraciones de nicotina por vía subcutánea y los sujetos tenían acceso a tres tipos de alimentos: alimento no dulce bajo en calorías, alimento dulce bajo en calorías y alimento dulce alto en calorías. Los resultados obtenidos mostraron que tanto el sabor dulce como el contenido calórico estuvieron involucrados en los efectos de la nicotina sobre el consumo de alimentos y el peso corporal, pues durante la administración de la droga el consumo de alimento dulce bajo en calorías fue preferido respecto al consumo registrado de los otros tipos de alimentos disponibles.

Grunberg, Popp y Winders (1988) examinaron los efectos de la nicotina en el peso corporal y el consumo de alimentos dulces y salados en ratas que tuvieron acceso a galletas, papas fritas, alimento de laboratorio y agua durante la administración de la droga. Como resultado obtuvieron que los sujetos que recibían nicotina consumieron menos galleta molida que las ratas que no recibieron nicotina. No se encontró ningún efecto de la nicotina en el consumo de comida de laboratorio o papas fritas. Después del cese de la administración de la droga, las ratas que recibieron nicotina mostraron relativamente tasas mayores de consumo de galletas que las ratas en los grupos control. En esta fase tampoco se presentaron los efectos de la nicotina en el consumo de comida de laboratorio o papas fritas.

Chen, Hansen, Jones, Vlahos, Anderson y Morris (2007) realizaron un experimento en ratones expuestos a la nicotina. Se les proporcionó alimento con altos niveles de grasa y descubrieron que la exposición a esta droga redujo el consumo de alimento por lo que la ingesta de calorías disminuyó en un 23\%. Al parecer esta exposición no aceleró el gasto de energía ni evitó la acumulación de grasa y la hiperglucemia.

De esta manera, la evidencia experimental previa sugiere que la nicotina tiene un efecto sobre la conducta alimentaria, en base a ello, el propósito de la presente investigación fue 
evaluar los efectos de la administración de nicotina sobre el peso corporal y la ingesta de alimento en ratas utilizando la aplicación de parches transdérmicos.

\section{Sujetos}

\section{MÉTODO}

Seis ratas de la sepa Wistar, tres machos y tres hembras experimentalmente ingenuas, con cuatro meses de edad al inicio del experimento, que estuvieron a libre acceso a alimento y agua.

Aparatos y materiales Se utilizaron seis cajas-habitación individuales, con medidas de $13 \mathrm{~cm}$ de altura por 27 cm de ancho y $38 \mathrm{~cm}$ de largo, con una reja metálica en la parte superior, con una división para comederos y bebederos. El fondo de la caja fue cubierto por una alfombra de aserrín, que se removió y sustituyó por otra cada cuatro días. Para el registro de consumo de alimento y peso corporal se utilizó una báscula electrónica de presión. Se proporcionó alimento de la marca Nutri-cubos, especial para animales de laboratorio, cuya fórmula nutricional es la siguiente: humedad $12 \%$, proteína $23 \%$, grasa $4.50 \%$, calcio $1 \%$, fibra 6\%, cenizas 8\%, ELN (extracto libre de nitrógeno) $46.50 \%$ y fosforo $0.61 \%$. Para beber se les proporcionó agua purificada.

La droga fue administrada por vía cutánea utilizando parches de nicotina, los cuales eran flexibles, de color piel y de forma rectangular, diseñados como un sistema transdérmico de liberación controlada y continua. La piel absorbe rápidamente la nicotina que se libera inicialmente del adhesivo del parche y pasa a la circulación general. Las concentraciones plasmáticas de nicotina alcanzan su punto máximo después de dos a cuatro horas de la aplicación inicial, con concentraciones en plasma relativamente constantes durante 24 horas (Alza Corporation, 2007).

\section{Procedimiento}

Los sujetos fueron identificados con un número, se registró el peso corporal al inicio del experimento y fueron colocados en cajas-habitación individuales. El peso corporal, el consumo de agua y comida se registró diariamente a las 8:00 de la mañana. Los sujetos fueron distribuidos en tres grupos: Grupo Experimental I, Grupo Experimental II y Grupo Control. Cada grupo se conformó de dos sujetos (una hembra y un macho). A las ratas del Grupo Experimental I se les colocó un parche de nicotina de $6 \mathrm{mg} / \mathrm{kg}$. A las ratas del Grupo Experimental II se les colocó un parche de nicotina de $12 \mathrm{mg} / \mathrm{kg}$. A las ratas del Grupo Control se les colocó un parche sin nicotina de las mismas características de los parches colocados en los otros dos grupos. La administración de la nicotina se realizó diariamente a la misma hora después del registro del peso corporal y el consumo de alimento. Para la aplicación de los parches se anestesió a cada sujeto (incluyendo a los sujetos del grupo control) por vía intraperitonial con un anestésico especial para cirugías en animales (pentobarbital sódico), la dosis que se administró fue de $30 \mathrm{mg} / \mathrm{kg}$ (dosis por debajo de la aconsejada para realizar cirugías mayores). A los diez minutos de administrada la sustancia se realizó la preparación del área a rasurar utilizando tijeras especiales para el corte de pelo, jabón en pastilla, agua potable y navaja para afeitar. Después de rasurar a los sujetos, éstos fueron regresados a sus cajas habitación en las que permanecieron 30 minutos, tiempo que tardan los sujetos en metabolizar la anestesia. Al 
término de este tiempo se colocó el parche, el área se preservó en condiciones asépticas idóneas durante las fases de manipulación.

Diseño experimental

El experimento se dividió en cinco fases. Las fases 1, 3 y 5 tuvieron una duración de diez días y los sujetos fueron expuestos a condiciones de libre acceso a alimento y agua. En las fases 2 y 4 se realizó la manipulación experimental: los sujetos tuvieron disponible alimento y agua en condiciones de libre acceso y se les administró la nicotina mediante la aplicación del parche transdérmico durante cinco días consecutivos.

\section{Resultados}

En las Figuras 1 y 2 se muestran los datos de registro diario del peso corporal y el consumo de alimento. Los puntos en color negro representan las condiciones de libre acceso a alimento y agua; los puntos de color gris representan las fases experimentales, en las que se administró la nicotina. En el panel superior se presentan el Grupo Experimental I, en el panel central se muestra al Grupo Experimental II y en la parte inferior de la figura se encuentra el Grupo Control. Los datos de los machos se presentan en la columna derecha y los datos de las hembras en la columna izquierda.

Respecto al peso corporal se observó que durante las fases experimentales los sujetos que estuvieron en contacto con la nicotina presentaron una disminución en su peso corporal en comparación con los periodos en los que no se les administro la droga, mientras que el Grupo Control no se observaron estas modificaciones. De manera particular, el sujeto ME 31 presentó una mayor disminución de peso corporal durante las fases experimentales, que posteriormente aumentó en las fases sin la droga.

El consumo de alimento por sujeto que se muestra en la Figura 2 muestra que durante las fases de manipulación se presentó un decremento en el consumo de alimento de los sujetos experimentales a diferencia de las fases sin nicotina, mientras que el consumo del Grupo Control no presentó modificaciones. Se observó que durante la Fase 2, el consumo de alimento disminuyó un promedio de $4 \mathrm{~g}$ en los grupos experimentales, mientras que durante la Fase 4 fue de $3 \mathrm{~g}$. Adicionalmente, puede observarse que el Grupo Experimental II registró los consumos de alimento más bajos durante la Fase 2, pues el sujeto ME 32 consumió 15 g durante el primer día de exposición a la nicotina, cuando su consumo promedio en línea base fue de 24 g. Por su parte, el sujeto HE 44 consumió sólo 6 g también durante el primer día de exposición, cuando su consumo promedio en línea base fue de $15 \mathrm{~g}$.

En las Figuras 3 y 4 se muestra el promedio del peso corporal y el consumo de alimento por grupos respectivamente. En la columna izquierda se presentan los promedios de los machos y en la columna derecha los promedios de las hembras obtenidos durante las cinco fases. Los puntos negros representan las fases sin acceso a la nicotina (1, 3 y 5) y los puntos en gris representan las fases con acceso a la nicotina (2 y 4).

En la Figura 3 puede observarse la disminución del peso corporal en los grupos experimentales cuando se administró la droga respecto a las fases sin la droga. No obstante, la disminución de peso corporal es más evidente en el caso de los machos que en de las hembras. Adicionalmente, no se observaron diferencias en la disminución del peso corporal entre los sujetos experimentales, es decir, entre los que recibieron 6 o $12 \mathrm{mg}$. Por otro lado, 
el macho control mostró una curva de crecimiento de peso corporal diferente a la de los grupos experimentales pues desde el inicio del experimento registró un peso corporal más alto respecto al de los otros sujetos.

En la Figura 4 se muestra el promedio del consumo de alimento de los sujetos durante las cinco fases. Se observó que los sujetos de los dos grupos experimentales mostraron una disminución en el consumo de alimento a partir de la administración de la droga, comparado con el consumo de alimento durante las fases 1, 3 y 5 . Esta disminución también se observa al comparar el promedio de consumo de alimento de los controles, quienes mantuvieron estable su consumo durante todo el experimento. De igual manera, la disminución en el consumo de alimento fue similar entre hembras y machos, sin observarse una diferencia entre los sujetos que fueron expuestos a 6 o $12 \mathrm{mg}$ de nicotina.

\section{Discusión}

Los resultados obtenidos mostraron que el peso corporal de los sujetos experimentales presentó una disminución durante las fases de administración de nicotina comparado con las fases sin aplicación de la droga y respecto al grupo control. También se observó la disminución del consumo de alimento cuando los sujetos experimentales fueron expuestos a la nicotina. Estos hallazgos son congruentes con los resultados obtenidos en estudios previos (Clarke \& Kumar 1984; Grunberg, Bowen, Maycock, \& Nespor 1985; Grunberg, Bowen, \& Morse 1984; Grunberg, Popp \& Winders 1987; Grunberg, Popp, \& Winders 1988). No obstante, en el presente estudio se utilizó como procedimiento para aplicar la nicotina el parche transdérmico, cuya técnica de administración es más sencilla y menos costosa que los otros procedimientos utilizados en las investigaciones previas.

La disminución en el consumo de alimento en los sujetos experimentales tiene otro punto de interés: la similitud en el promedio de consumo de alimento registrado a partir de la aplicación del parche de nicotina de $6 \mathrm{mg}$ respecto al de $12 \mathrm{mg}$. Grunberg, Bowen y Morse (1984) utilizaron en sus experimentos dosis de nicotina de 4, 8 y $12 \mathrm{mg}$ y obtuvieron resultados similares: no encontraron diferencias significativas en el promedio de consumo de alimento registrado entre los grupos que recibieron estas dosis. Al parecer, la administración de la droga disminuye el consumo de alimento independientemente de la dosis utilizada. Posiblemente, la aplicación de dosis más fuertes podría mostrar resultados más contundentes.

Otras investigaciones previas concluyeron que cuando se presentan alimentos con alto contenido calórico la rata presenta un mayor consumo de estos alimentos y que en las fases de administración de nicotina este consumo disminuye. Asimismo, cuando se suspende la administración de la droga el consumo de los alimentos altos en calorías se presenta como en la fase inicial (Grunberg, Bowen, Maycock \& Nespor 1985). De esta manera, se sugiere que en investigaciones posteriores en las que se administre nicotina a través de parches transdérmincos se considere también el registro del consumo de calorías, antes, durante y después del procedimiento experimental. De igual forma, se sugiere variar el tipo de alimentos disponibles en cuanto a sabor, textura y contenido energético. Es probable que el resultado de estas manipulaciones muestre efectos diferenciales de la nicotina sobre la conducta alimentaria.

Respecto al consumo de alimento registrado para hembras y machos pueden notarse algunas diferencias: los machos mostraron una disminución mayor que las hembras, de 
igual manera, en las etapas donde se suspendió la administración de la nicotina, el aumento en el consumo de alimento fue mayor en los machos experimentales. Estos resultados no coinciden con los que reportan Grunberg, Winders y Popp (1987) quienes afirmaron que la administración de nicotina y su suspensión tiene mayores efectos en el peso corporal y patrones alimentarios de las hembras a diferencia de los machos. Para esta generalización se basaron en estudios del peso corporal y hábitos de alimentación en periodos de dos a tres semanas antes, durante y después de la administración de nicotina, la cual fue administrada durante 16 días por medio de bombas mini osmóticas. Por consiguiente, concluyeron que las diferencias sexuales pueden reflejarse en la sensibilidad a la nicotina. No obstante, los resultados obtenidos en el presente trabajo plantean otras interrogantes relacionadas al género en relación al tiempo en el que la sustancia estuvo disponible, así como a las dosis utilizadas. Por lo que es importante realizar más investigaciones que utilicen mayor cantidad de sujetos para comprobar si esta diferencia es significativa.

Por otro lado, se sugiere que los resultados obtenidos en el presente trabajo difieren de los resultados obtenidos en estudios previos en los que las diferencias en el consumo de alimento a partir de la administración de la nicotina es más evidente debido a la técnica utilizada. En trabajos anteriores se utilizó la administración directa en sangre, mientras que en el presente trabajo se hizo a nivel transdérmico. Entre las ventajas de esta técnica se encuentran su fácil de utilización, costo y menor grado de invasión al organismo: no causa daños físicos y disminuye el grado de estrés en los sujetos. Sin embargo, una de sus desventajas es la posibilidad de causar daños en la piel. Los parches transdérmicos están diseñados para la utilización en piel de características muy particulares, como la del ser humano, por lo que al utilizarla en la piel de animales, como por ejemplo en ratas, se presentan problemas para que se mantengan adheridos al sujeto durante periodos de tiempo más prolongados.

Por otro lado, los resultados de este trabajo muestran otro punto de interés: el consumo de nicotina puede ayudar a mejorar el mantenimiento del peso corporal adecuado. Otras investigaciones también han demostrado otras ventajas del consumo de nicotina. Al parecer, la administración de nicotina por cualquier vía tiene un efecto positivo sobre algunos procesos cognitivos como por ejemplo: la memoria, ejecuciones psicomotoras y en general sobre los procesos atencionales. En distintos estudios se han obtenido como resultado que los fumadores mejoran el procesamiento de la información y que en animales facilita la ejecución de situaciones que implique memoria y aprendizaje (Redolat, Carrasco y Simón, 1994).

La nicotina también se ha utilizado como tratamiento a pacientes con enfermedades como el Alzheimer, White y Levin (1999) realizaron un estudio en el que administraron nicotina por vía trasdérmica durante ocho semanas con descansos intermedios de dos semanas a pacientes diagnosticados con la enfermedad de Alzheimer en el nivel de leve a moderado. A dichos voluntarios se les colocó un parche de nicotina durante dieciséis horas diarias, la primera semana se les administró $5 \mathrm{mg}$, la segunda y tercera semanas se les administró 10 mg y durante la cuarta semana se les administró 5 mg. Durante el experimento se les realizaron evaluaciones de memoria y atención, como resultado obtuvieron que la nicotina mejoró significativamente el desempeño de la atención, medido por la prueba de funcionamiento continuo de Conners, sin embargo, la nicotina no mejoró el rendimiento en las pruebas de memoria. 
En otros estudios experimentales, el consumo de nicotina ha ayudado a mejorar la atención y la percepción, sin embargo, es bien conocido que la nicotina es una sustancia altamente adictiva. Esto se ha demostrado en situaciones de laboratorio con animales que se autoadministran la droga. El animal es expuesto a un programa de reforzamiento de razón fija y se le coloca a los sujetos un catéter intravenoso. El sujeto es entrenado para que se realice la autoadministración de la sustancia de acuerdo a un esquema de refuerzo: para que la sustancia sea administrada es necesario que el animal realice un palanqueo. En este tipo de estudios se demuestra que el animal realiza las tareas que sean necesarias para obtener los efectos que esta sustancia provoca en su organismo (Granda, Solano, Jareño, Pérez, Barrueco y Jiménez 2006).

De igual manera, como es bien conocido, la administración de nicotina tiene un efecto negativo sobre la salud de sus consumidores, por ejemplo se ha demostrado que el consumo de esta sustancia está relacionado con algunos tipos de cáncer y enfermedades coronarias. En resumen los resultados obtenidos en estudios de laboratorio sugieren que la nicotina puede ayudar a mejorar los procesos atencionales, sin embargo también se ha demostrado que esta sustancia puede provocar daños severos al organismo. A partir de esta evidencia se abren las siguientes interrogantes $\dot{i}$ El consumo de la nicotina se considera positivo para lograr una mejora en el aprendizaje?, ¿Es recomendable que pacientes con déficit de atención consuman nicotina?, ¿La administración de nicotina se debe considerar como un tratamiento a pacientes con enfermedades como el Alzheimer, a pesar de los efectos negativos que esta sustancia provoca?. Finalmente ¿la nicotina puede convertirse en una solución alterna ante los problemas de sobrepeso de la población? Sin duda los argumentos a favor y en contra del consumo de esta droga son ampliamente discutibles. Es evidente que no es posible dar una respuesta certera ante estos cuestionamientos. Sin embargo, pareciera que sus efectos negativos son más determinantes que sus efectos positivos. Por tanto, es necesario seguir estudiando los efectos que esta sustancia tiene sobre el organismo y su conducta, sobre todo durante periodos de administración prolongados.

\section{Referencias}

Alza Corporation. (2007) NIQÜITIN. [En línea]. Recuperado el día: 07 de Noviembre de 2008 disponible en:http://www.libreriamedica8a.com/productos/2186.htm

Azevedo, P., Minicucci, M., Matsubara, B., Matsubara, L., Duarte, D., Paiva, S. y Zornoff, M. (2010). Estándar de remodelación y función ventricular en ratones expuestos al humo del cigarrillo. Arquivos Brasileiros de Cardiología, 94, 212-215.

Barber, E., Fox, M., Roca, G. y Fresneda, M. (1987). Efectos sobre el crecimiento renal de ratas normales de la administración intraperitoneal de urea. Revista Cubana de Investigaciones Biomédicas, 6, 7-16.

Chen, H., Hansen, M., Jones, J., Vlahos, R., Anderson, G. y Morris, M . (2007) Detrimental metabolic effects of combining long-term cigarette smoke exposure and high-fat diet in mice. American Journal of Physiology: Endocrinology and Metabolism, 293, E1564E1571. 
Clarke, P. y Kumar, R. (1984). Some effects of nicotine on food and water intake in undeprived rats. British Journal of Pharmacoogy, 82, 233-239.

Genn, R., Tucci, S., Edwards, J. y File, S. (2003). Dietary restriction and nicotine can reduce anxiety in female rats. Neuropsychopharmacology. 28, 1257-1263.

Granda, J., Solano, S., Jareño, J., Pérez, A., Barrueco, M. y Jiménez, C. (2006). De la neurobiología de la adicción a la nicotina al tratamiento del tabaquismo. Progresos terapéuticos. Prevención del Tabaquismo, 8, 116-128.

Grunberg, N., Bowen, D. y Morse, D. (1984). Effects of nicotine on body weight and food consumption in rats. Psychopharmacology, 83, 93-98.

Grunberg, N., Bowen, V., Maycock, D. y Nespor, S. (1985). The importance of sweet taste and caloric content in the effects of nicotine on specific food consumption.

Psychopharrnacology, 87, 198-203.

Grunberg, N., Winders, S. y Popp, K. (1987). Sex differences in nicotine's effects on consummatory behavior and body weight in rats. Psychopharmacology, 91, 221-225.

Grunberg, N., Popp, K, y Winders, S. (1988). Effects of nicotine on body weight in rats with access to "junk" foods. Psychopharmacology, 94, 536-539.

Jiménez- Ruiz, C., Ayesta, J., Planchuelo M., Abengozar, R., Torrecilla M., Quintas A., Hernández, M., De la Cruz, E., Ramos, B., Romero, J., Alonso, S., De Granda, I., Méndez, C,. Camarelles, F., Solano, S. y Grupo SEDET. (2001).

Recomendaciones de la Sociedad Española de Especialistas en Tabaquismo (SEDET) sobre el tratamiento farmacológico del tabaquismo. Prevención del Tabaquismo, 3, 30-38.

Nuutinen, S (2007). The effects of nicotine on the regulation of neuronal nicotinic acetylcholine receptors and intracellular signalling pathways. [En línea]. Recuperado el día: 09 de Diciembre de 2009. Disponible en: https://oa.doria.fi/dspace/bitstream/10024/4047/1/theeffec.pdf

Redolat, R.,Carrasco, M. y Simón, V. (1994). Efectos cognitivos de la nicotina y el tabaco en sujetos humanos. Psicothema, 6, 5-20.

Salin-Pascual, R., De la Fuente, J., Galicia-Polo, L. y Drucker-Colin, R. (1995). Effects of transderman nicotine on mood and sleep in nonsmoking major depresssed patients.

Psychopharmacology, 121, 476-479.

White, H. y Levin, E. (1999). Four-week nicotine skin patch treatment effects on cognitive performance in Alzheimer’s disease. Psychopharmacology, 143, 158-165. 
Peso corporal

Grupo Experimental I
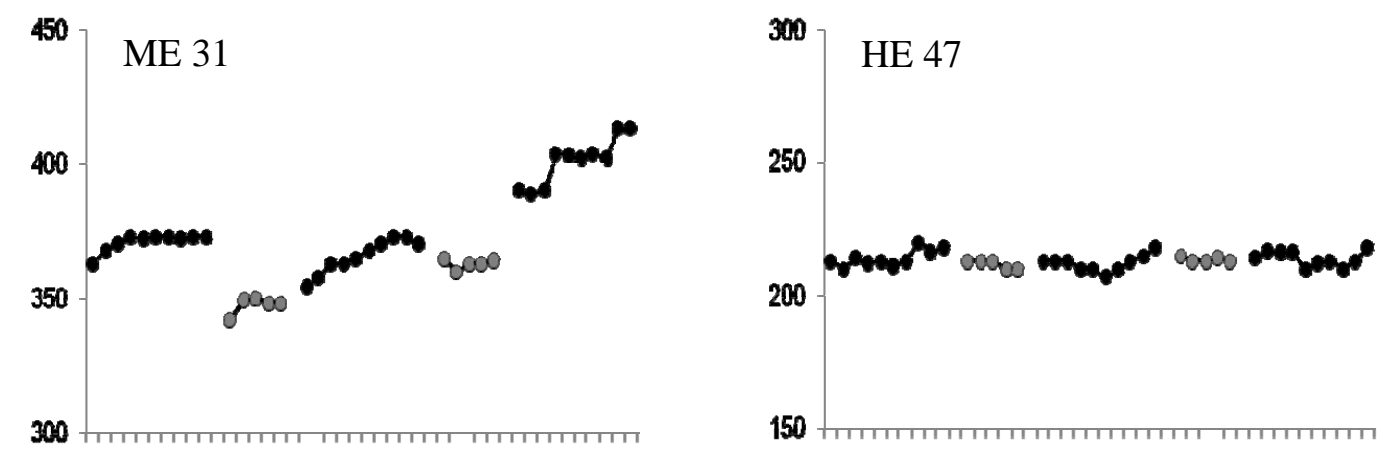

Grupo Experimental II
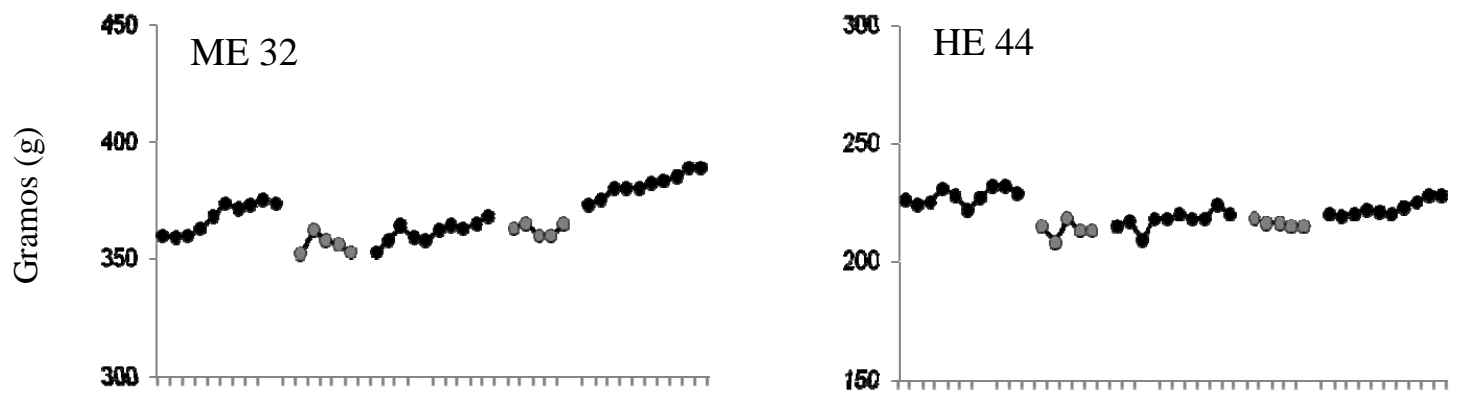

\section{Grupo Control}
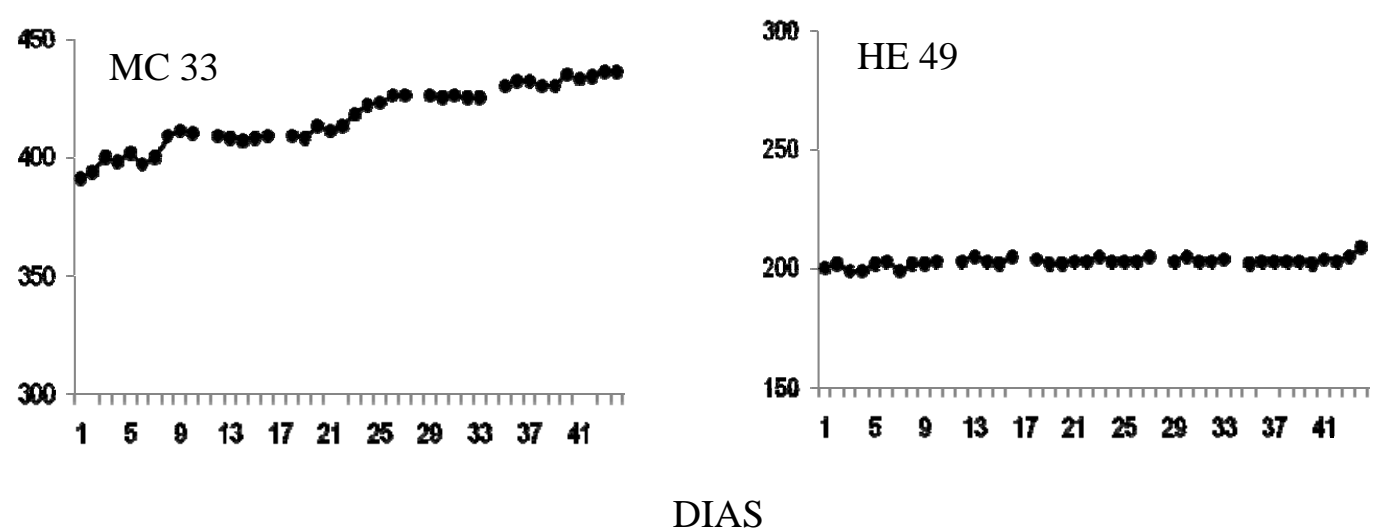

Figura 1. Peso corporal. El panel superior muestra los datos del Grupo Experimental I, el central representa el grupo Experimental II y el panel inferior muestra el Grupo Control. Se presentan los datos de los machos y hembras en las columnas izquierda y derecha respectivamente. Los puntos negros representan las fases sin nicotina y los puntos grises muestran las fases con nicotina. 
Consumo de alimento

Grupo Experimental I
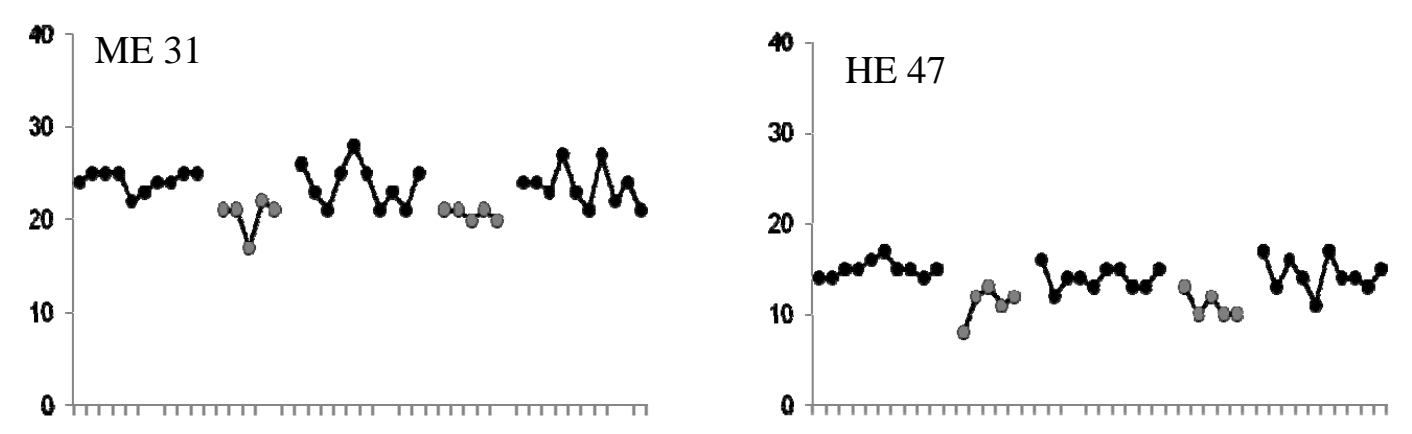

Grupo Experimental II
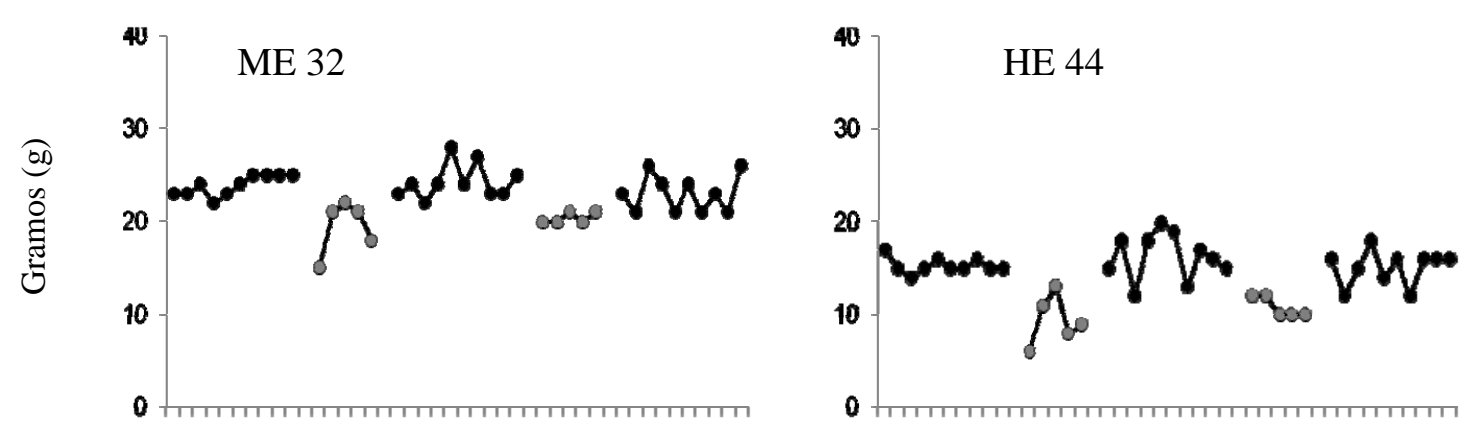

Grupo Control

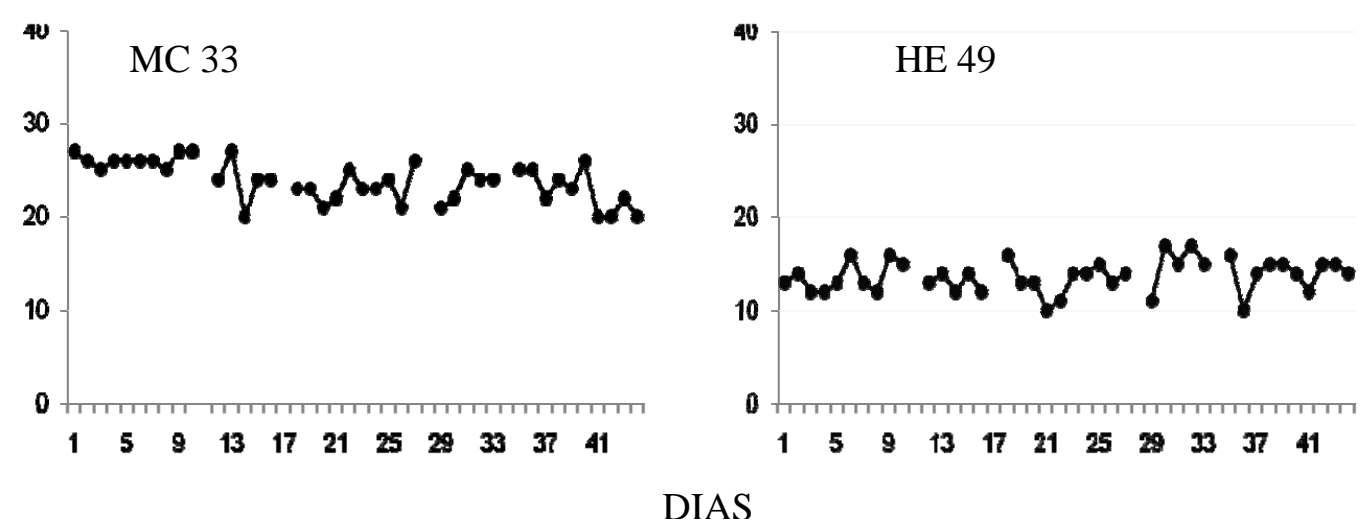

Figura 2. Consumo de alimento. El panel superior muestra los datos del Grupo Experimental I, el central representa el Grupo Experimental II y el panel inferior muestra el Grupo Control. Se presentan los datos de los machos y hembras en las columnas izquierda y derecha respectivamente. Los puntos negros representan las fases sin nicotina y los puntos grises muestran las fases sin nicotina. 
Promedio del peso corporal por grupos
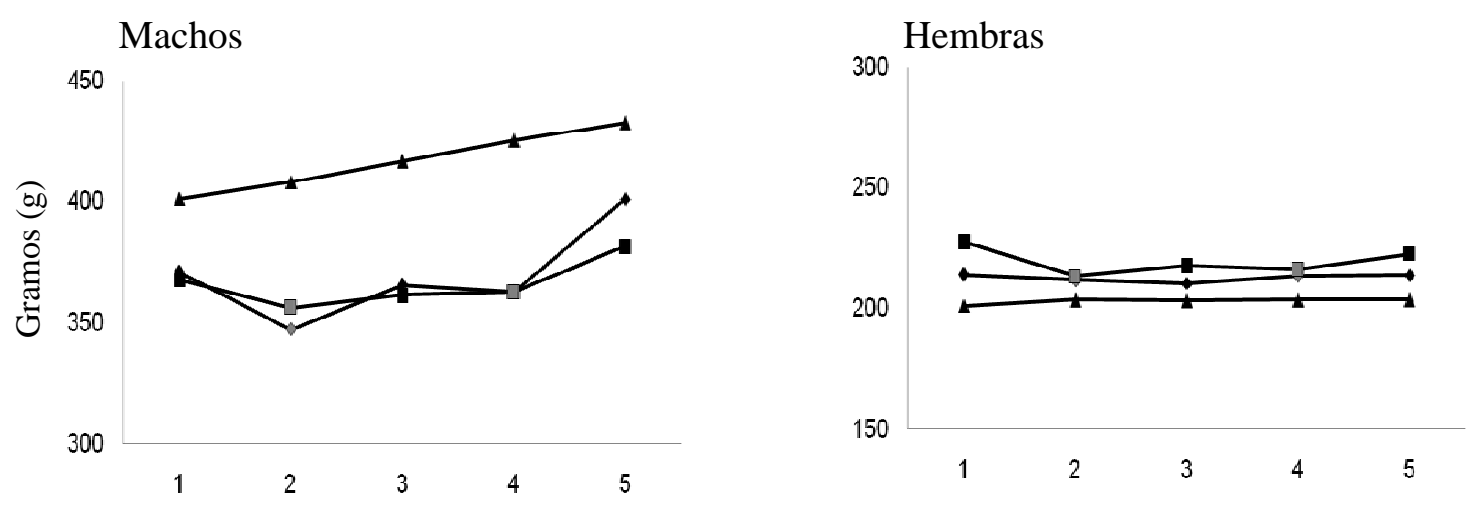

Fases

Figura 3. Peso corporal. En la parte izquierda de la figura se presenta el promedio del peso corporal registrado de los machos y en la columna derecha de la figura se muestra el promedio del peso corporal de las hembras obtenido durante las cinco fases. Los $\boldsymbol{\Delta}$ representan el Grupo Control, $\diamond$ representa el Grupo Experimental I (6mg.) $\diamond$ representa la fase experimental y $\boldsymbol{\square}$ representa el Grupo Experimental II (12mg.) y $\square$ representa la fase experimental. 
Promedio del consumo de alimento por grupos
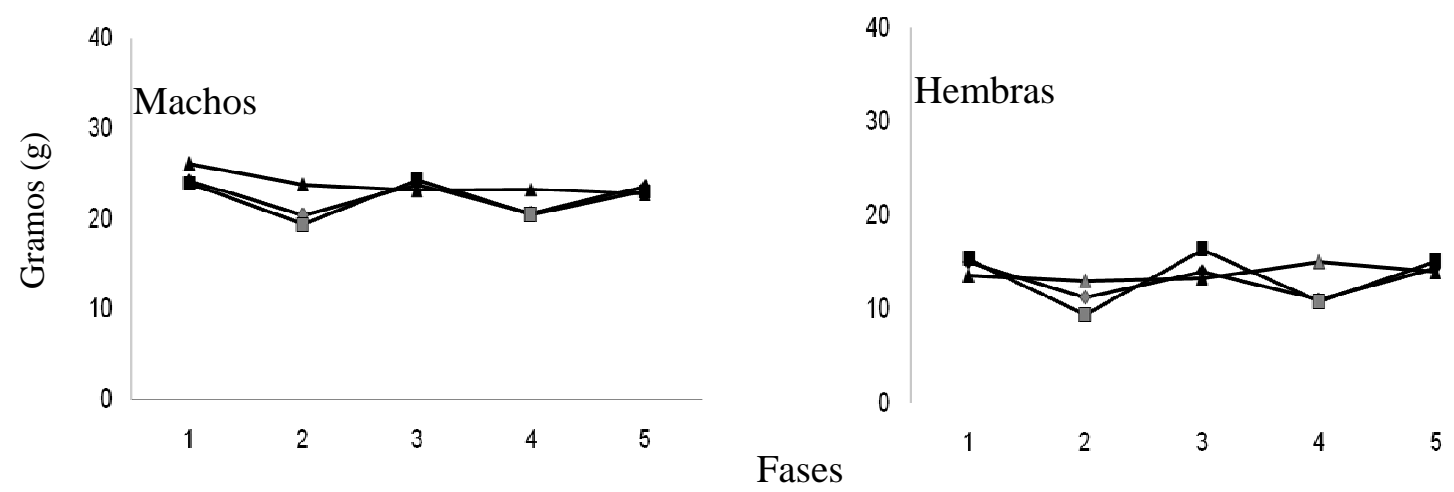

Figura 4. Consumo de alimento. En la parte izquierda de la figura se presenta el promedio del consumo de alimento de los machos y en la columna derecha de la figura se muestra el promedio del consumo de alimento de las hembras obtenidos durante las cinco fases. Los $\Delta$ representan el Grupo Control, $\diamond$ representa el Grupo Experimental I (6mg.) $\diamond$ representa la fase experimental y $\square$ representa el Grupo Experimental II (12mg.) y $\square$ representa la fase experimental. 